\title{
Using a Novel Blending Method Over Multiple Network Connections for Secure Communication
}

\author{
Jaime C. Acosta \\ U.S. Army Research Laboratory \\ White Sands Missile Range, NM 88002-5513 \\ http://www.jaimeacosta.info/
}

\author{
John D. Medrano \\ U.S. Army Research Laboratory \\ White Sands Missile Range, NM 88002-5513
}

\begin{abstract}
In the field of computer security, covert communication is usually seen as adversarial, but from another perspective, it can be seen as a way to communicate securely by hiding data from a malicious third party, e.g., an inside attacker. In this light, instead of making data unreadable using encryption, it may be possible to hide from an adversary a secure network infrastructure (consisting of several node endpoints) in network traffic.

In this paper we describe a novel blending technique that is capable of using as carriers the payload fields of multiple connections including audio, video, and voice over IP (VoIP) streams. The technique executes in three main phases. First the state of the network is analyzed. Next, insertion points are selected based on the protocols, data rates, and randomness characteristics of the network data. Finally, covert data are inserted into packets that are injected into the network. By analyzing the same network traffic the covert receiver identifies the insertion points and extracts the covert messages. In this paper, we evaluate the blending covert method with user datagram protocol (UDP) connections during two network loads. Our results show that our technique works with limited data loss. We also provide an analysis of the trade offs between throughput and detectability.
\end{abstract}

\section{INTRODUCTION}

Much focus has been placed on security defense mechanisms against outside adversaries, but an insider with malicious intent can overcome these defenses with much less effort. One example of an inside attack is privilege escalation. In a network environment with promiscuous traffic such as a wireless local area network (WLAN), an inside attacker may eavesdrop on communication to realize a network's infrastructure. Although some of these networks employ encryption, many encryption techniques allow an insider to see all traffic on the network. In this case, an insider could use this information to plan an effective denial of service attack. Similar scenarios are possible in networks with multicast traffic, hubs, or switches (e.g., using a poisoning attack). One way to protect data from unintended receivers is to hide communication by using covert channels.

Traditionally, covert communication is defined as a way for two entities that are not intended to communicate to exchange information [1]. From this perspective, covert communication is adversarial, and is used as a means to leak information to unintended listeners. From a different perspective, covert communication can be seen as a way to protect information from unauthorized recipients. Unlike encryption, where the information is made unreadable, covert communication will allow hiding information including node endpoints in a network environment. This will make it difficult for a malicious insider to plan attacks.

Some have used covert communication for legitimate communication, e.g., [2]-[4], but in general, these covert communication methods are tied to a single protocol and throughput does not scale as the network gets larger. In this paper, we introduce the blending covert method (BCM), which allows nodes in a network to communicate covertly by blending in active network traffic.

In this paper, we make the following contributions:

1) We describe a method that can be used for communicating securely through multiple concurrent covert channels within a network with promiscuous traffic (e.g., WLAN, switch with port mirroring, hub, and multicast). The covert throughput scales as network congestion increases.

2) We evaluate the performance of the BCM under two loads and show that the method works with limited data loss in a hub network. We also evaluate BCM with different parameter configurations to show the trade offs between throughput and detectability.

The rest of the paper is organized as follows. First, we review related covert communication methods. Next, we describe the covert communication method and each phase (monitor, select, and insert/extract) in detail along with the configurable parameters. We then describe the experimental setup and report the results from the experiments. Finally, we present our conclusions and future work.

\section{RELATED WORK}

There are two main types of channels that are used for covert communication [5]. The first type are timing channels, which work by purposely modifying timing mechanisms on a network such as packet arrival times to encode data. In general, timing channels are difficult to detect, but they provide low throughput. The second type are storage channels, which insert covert data inside header or footer fields in specific protocols, e.g., [6], [7] and within payload fields of invalid messages. For example, HICCUPS [8] works by hiding data within the payload of messages with bad checksums in the datalink layer. This method works for wireless networks and 
requires specialized hardware that has the capability to modify data link layer checksums. In general, once these channels are documented, an adversary may know where to find these.

Recent methods use a combination of timing and storage channels. The Lost Audio PaCKets Steganography (LACK) method [9] works by having a legitimate voice over IP (VoIP) stream between nodes on a network. The sender will then hide data in purposely delayed packets. In a VoIP stream messages older than a certain period are usually ignored because each message generally carries only a few milliseconds of audio. It would not make sense for a receiver to process older messages, especially in streams with high message rates. Only a covert receiver captures and extracts the covert data within these messages. From a defensive perspective, just as a covert receiver monitors the old messages an adversary could potentially do the same and therefore breach the covert communication.

One possible solution for this problem is to blend covert communication with normal traffic. In the field of system vulnerability analysis, one method that uses blending to bypass anomaly-based intrusion detection systems (IDSs) is the polymorphic blending attack (PBA) [10]. PBA works by monitoring traffic and identifying byte frequencies. In order to send malicious packets without triggering an anomaly detector, PBA pads the malicious data with bytes that make the entire contents match known byte frequencies.

Applying this blending idea to covert communication requires additional considerations. First, covert communication requires a sender and a receiver, so the data must be coded in a way that a receiver can identify and extract the messages. In addition, assuming that invalid messages are not ignored, a covert communicator should modify only portions of messages that will not be noticed. Yarochkin et al. [11] demonstrate a method that creates connections that blend with network traffic (including protocols and services used). These connections are used to communicate covertly and they are created and removed dynamically as the network changes. The method relies on having a network structure where new connection attempts are common and failed connection attempts are blocked and ignored, such as in Internet traffic. This is not the case in, e.g. tactical networks where all nodes and connections among nodes follow some specifications.

Huang et al. [12] present another method that blends covert communication with existing traffic by hiding information in VoIP streams. In this work, the authors use the least significant bits (LSBs) in VoIP streams to hide data. The LSBs are used regardless of the data that is being sent. One problem that can occur is if the LSBs are unchanging, when inserting covert data the covert communication may become obvious. An extension of this work [13], improved the covert insertion process by selecting the LSB most similar to a given covert message to improve the blending (or transparency). The latter two methods use a single protocol and use fixed fields in the payload for covert communication. The method described in this paper chooses insertion points dynamically and embeds covert data over multiple connections.

\section{Blending Covert Method}

The blending covert method works in three main phases. During each phase, there are several parameters that a covert communicator may set in order to balance between throughput and detectability, e.g., one may risk higher detectability to achieve higher throughput in a network with some level of congestion depending on the protocols and services used.

The three phases and the parameters used are shown in Figure 1.

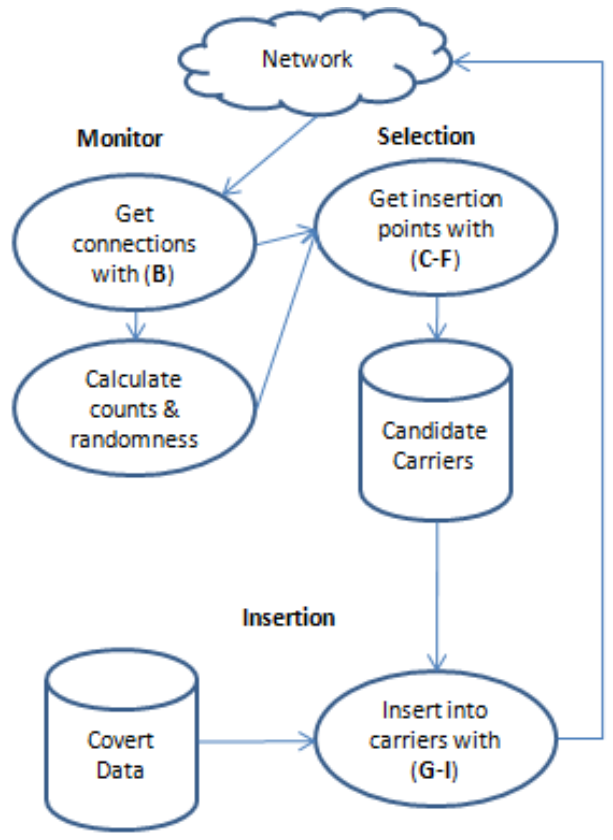

Fig. 1. Blending covert method dataflow diagram. Letters in parentheses correspond with the parameters in Table I.

\section{A. Monitoring}

In the monitor phase, all network traffic is analyzed in promiscuous mode. A connection consists of the messages sent between two nodes that share the same source and destination media access control (MAC) addresses, Internet protocol (IP) addresses, and port numbers. In addition, messages in a connection have equal protocol types and packet lengths. Therefore, for each connection, only the bytes in the payload fields change.

In this phase, connections that communicate over the protocol specified in the Protocol to Use parameter are stored. For each of these connections, several statistics are calculated. First, the rate is calculated by averaging the number of packets received during the last two windows of duration given by Window Size. Next, the randomness of the each byte in the connection and the total over the entire connection are calculated. Randomness is calculated as follows.

Using the packets collected for a given connection, the randomness of each byte in the buffer is calculated using a histogram. Given that each byte in the buffer can take one of 256 possible values, a histogram is generated using the 
TABLE I

Blending Covert Method Parameters

\begin{tabular}{|c|c|c|c|}
\hline Type & Parameter Name & Description & Value Range \\
\hline Monitor & (A) Window Size & $\begin{array}{l}\text { Determines how often the packet counts, byte counts, and ran- } \\
\text { domness values are computed. Also defines the smallest wait time } \\
\text { before sending a covert message for a connection. For example, } \\
\text { if this value is set to one second, for each connection, only one } \\
\text { covert message can be sent each second. }\end{array}$ & {$[100 \mathrm{~ms}, 5000 \mathrm{~ms}]$} \\
\hline Monitor & (B) Protocol To Use & $\begin{array}{l}\text { Indicates which network protocol(s) must be used by a connection } \\
\text { to be a candidate for covert message insertion. }\end{array}$ & [UDP,any] \\
\hline Selection & (C) Rate Threshold & $\begin{array}{l}\text { The minimum rate (packets per window) that a connection must } \\
\text { send data to be a candidate for covert data insertion. A higher } \\
\text { value will decrease detectability, but will also reduce throughput. }\end{array}$ & {$[1,50]$} \\
\hline Selection & (D) Connection Randomness Threshold & $\begin{array}{l}\text { This value is calculated by summing the randomness of all bytes } \\
\text { in a connection. This value indicates the minimum randomness } \\
\text { that a connection must exhibit to be a candidate for covert data } \\
\text { insertion. }\end{array}$ & {$[1,1024]$} \\
\hline Selection & (E) Byte Randomness Threshold & $\begin{array}{l}\text { Byte randomness is calculated using the technique described in } \\
\text { Section III-A. This value indicates the minimum randomness that } \\
\text { a byte in a connection must exhibit to be a candidate for covert } \\
\text { data insertion. }\end{array}$ & {$[0,1]$} \\
\hline Selection & (F) Contiguous Random Bytes & $\begin{array}{l}\text { Indicates the number of contiguous bytes that must satisfy the } \\
\text { Byte Randomness Threshold in order for a connection to be a } \\
\text { candidate for covert data insertion. }\end{array}$ & $\begin{array}{l}{[\text { Sync Bytes }+} \\
\text { Checksum Bytes } \\
+1,1024]\end{array}$ \\
\hline Insertion/Extraction & (G) Sync Bytes & $\begin{array}{l}\text { Indicates the number of bytes to use for identifying the start of } \\
\text { a covert message within a connection's payload. A higher sync } \\
\text { byte count will result in fewer false positives, but may also result } \\
\text { in higher detectability and less throughput. }\end{array}$ & {$[0,5]$} \\
\hline Insertion/Extraction & (H) Checksum Bytes & $\begin{array}{l}\text { Indicates the number of bytes to use for calculating the message } \\
\text { checksum used to validate covert messages. A higher value will } \\
\text { result in fewer false positives, but less throughput. }\end{array}$ & {$[0,3]$} \\
\hline Insertion/Extraction & (I) Rate To Use & $\begin{array}{l}\text { Indicates the percentage of the connection's rate that will be used } \\
\text { for covert data insertion. }\end{array}$ & {$[0,1]$} \\
\hline
\end{tabular}

number of occurrences of values or range of values for each byte. Figure 2 shows sample histograms for three cases. In these cases, the value ranges for the bytes are stored in eight bins (x-axis). Each time a new packet is received, the bin corresponding to the byte value is incremented (y-axis). The leftmost histogram is for a byte that exhibits a predominate value with some occurrences of surrounding values. The middle histogram shows a byte value that is mostly evenly distributed (which is most favored for covert data placement), while the rightmost graph shows a byte value that has three discrete value ranges.

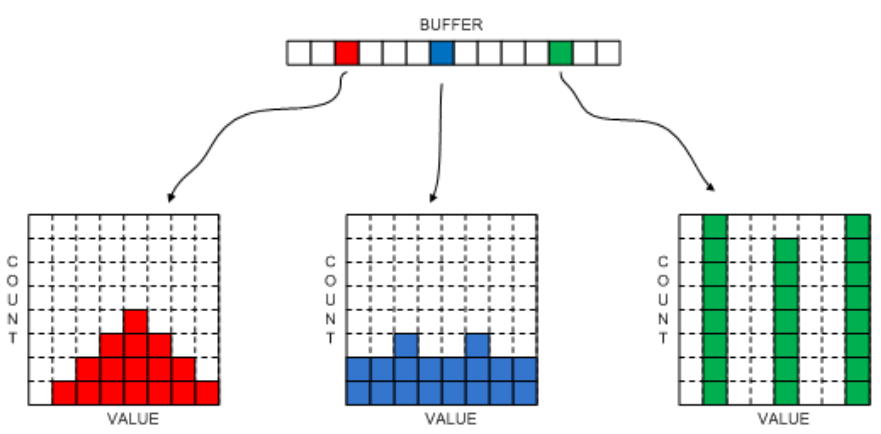

Fig. 2. Sample histograms for different randomness values

For this work, we used histograms with equal size bins with width size of 32 (8 bins) in order to produce a good estimate of the probability density function (PDF) [14]. This was verified with informal testing. A histogram with evenly distributed value counts produces a uniform PDF.

Given that the works in real time, we calculated a single value representation of randomness for each byte using a method that requires minimal resources. We used the following equation.

$$
R_{b}=\operatorname{Min} / \operatorname{Max}
$$

Min and Max represent the minimum and maximum count values of the histogram. If the values are well distributed then the maximum and minimum values will be close in value and the randomness will be close to one. Higher counts in discrete bins will result in decreased randomness. In the case where a bin has a count of zero, the resulting randomness will be zero and the byte will not be considered for data insertion. This is because communication protocols usually designate fixed values for certain bytes to indicate status, flags, and control. When inserting covert data, these bytes may be overwritten with illegal values that may alert a third party.

For a given connection, the total randomness is given by the following.

$$
\sum_{b=1}^{n} R_{b}
$$


where $n$ is the size of a connection.

\section{B. Selection}

During the selection phase, locations for placing covert data are identified and stored. Based on the counts from the first phase, in order to blend with active network traffic, connections that satisfy the Rate Threshold, and the Connection Randomness Threshold are selected. Within these connections, the sequences of contiguous bytes satisfying the parameters Contiguous Randomness Bytes and Byte Randomness Threshold) are identified. Next, the start and end position of the longest sequence, along with a copy of the packet is stored as a candidate carrier.

\section{Insertion and Extraction}

The insertion phase runs on a separate thread that continually pulls covert data from a buffer. This buffer is filled by reading the contents of a file. If candidate carriers exist, then covert data are distributed among the candidate carriers into the insertion points. Along with the covert data, sync bytes and checksum bytes are also included in the sent data. The number of sync and checksum bytes used are taken from the Sync Bytes and Checksum Bytes parameters. In the case that candidate carriers do not exist in the current window, then the data are held in the buffer. When a message is sent, the candidate carriers that are used will not be considered again for a specific number of windows, given by the Rate to Use parameter.

One important note is that due to the header fields exhibiting zero randomness (because they never change for a connection), these fields are not considered as insertion points. Therefore, the covert data are sent in packets without modification to the source, destination and protocol fields.

During the extraction phase, a covert receiver extracts covert data by monitoring the network traffic and looking for sync bytes and valid checksums. Assuming a covert sender uses insertion points that exhibit uniform PDFs, then an estimate of the chance of a false positive is given by the following.

$$
P_{\text {FalsePositive }} \approx(1 / 256)^{n}
$$

One way to reduce the number of packets analyzed by a receiver is to match the parameters used by the sender (see Table I). However, this may result in higher packet loss if the parameters are too strict.

\section{EVALUATION}

In order to test the performance of the BCM, we implemented a tool and tested it in real network environment. The tool is written in the Java programming language and uses the open source JNetPcap library for network operations.

\section{A. Experimental Setup}

We evaluated our covert method by measuring throughput, reliability, and detectability under different network loads. We evaluate reliability by measuring packet loss and then we evaluate trade offs between throughput and detectability by testing the method using different parameters values.
For the first experiment, the network setup contains eight nodes connected on a $100 \mathrm{mbps}$ network hub. One node is the covert sender, one node is the covert receiver and the other six nodes are overt communicators that exchange mp3 audio using the real time service protocol (RTSP) enclosed in UDP. In total there are three pairs of connections among the six nodes. Each node acts as a server and client to a neighboring node (i.e. node A serves node B and node B also serves node A, etc.). The publicly available live555 media server software is used to serve the audio and the video lan client (VLC) tool is used to play the audio. Each node serves a different mp3 file (a total of six different mp3 files are used). The audio files are set to loop in order to keep a continuous data stream. The mp3 audio files are recorded conversations taken from a dialog corpus [15].

In order to observe the effects of the network load on throughput (specifically packet loss), we conducted a second experiment with a higher amount of network congestion. For the second experiment, the network setup consisted of the same eight nodes, plus six additional nodes exchanging transmission control protocol (TCP) packets. We used a traffic generator to generate the TCP traffic, which was mostly audio and video data.

Table II shows the network load conditions during both experiments.

TABLE II

NETWORK TRAFFIC LOADS DURING EXPERIMENT 1 (CENTER COLUMN) AND EXPERIMENT 2 (RIGHTMOST COLUMN)

\begin{tabular}{|l||r|r|}
\hline & Load A & Load B \\
\hline Overt Nodes & 6 & 12 \\
\hline Packets/sec & $80-100$ & $5200-5500$ \\
\hline Bytes/sec & $15-20$ & $40-50$ \\
\hline Active Connections & $95,000-115,000$ & $2,700,000-3,500,000$ \\
\hline
\end{tabular}

In both experiments, only the UDP connections were used as carriers for covert communication. The reason UDP was used is because the protocol is connectionless and there is no control mechanism as there is in TCP (with sequence numbers). During the send phase, only payload data are modified, not header fields. Duplicating TCP messages could alert an adversary to the covert communication. One way that TCP could be used is if a covert sender is also a legitimate sender that embeds covert data in the payload of select messages.

As the experimental control, the following parameter values were used. We used two sync bytes and two checksum bytes to virtually eliminate false positives. The other parameters were chosen favoring detectability over throughput.

- Window Size $=1000 \mathrm{~ms}$

- Sync Bytes = 2

- Checksum Bytes = 2

- Rate to Use $=0.1$

- Protocol to Use = UDP

- Rate Threshold $=10$

- Connection Randomness Threshold $=10$

- Contiguous Random Byte $=(1+3)+1=4$ 
We ran each experiment with varying values for the Byte Randomness Threshold parameter and we measured the throughput and reliability during each run.

For each run, we first started a covert sender, which would automatically begin sending covert packets when valid threshold values were observed. Each packet included a sequence number (0-255) as the first byte of data. The sequence numbers were used to identify lost packets. On the receiver, missing or out-of-order sequences indicated missing packets.

On the sender, a large file was loaded into the covert data buffer. As a result, the buffer always contained enough data to fill all candidate carriers during each window. This was done to measure maximum throughput.

The receiver was started fifteen seconds after the covert sender. Packet loss was observed only after the first packet was received. The receiver was run for five minutes, during which all incoming covert messages were logged. After the experiment, we analyzed the logs to measured throughput and packet loss.

\section{RESULTS}

We measured throughput using different Byte Randomness Threshold values (to represent detectability levels). Figure 3 shows these results. The amount of throughput is consistent under both network loads (because the same UDP traffic was present for both). Also, as the threshold value is increased, the throughput decreases, but this also means that the data are placed in areas with higher randomness, which would be more difficult for an adversary to find. It should be noted that when using a value of 0.9 for the Byte Randomness Threshold, no covert data were sent. Although there were some individual bytes that exhibited this high randomness, there were not enough contiguous bytes to fit the covert data.

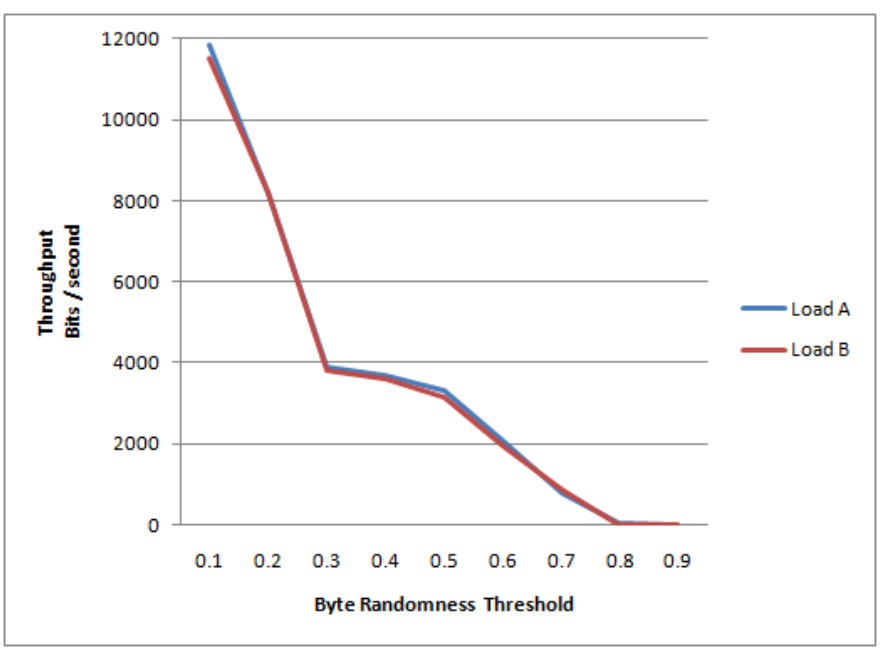

Fig. 3. Throughput graphed against Byte Rate Thresholds

We measured reliability by counting the misses associated with the throughput presented in Figure 4. Figure 4 shows these results. The data points are the total number of misses averaged over the five minute runs. Even using UDP connections, which are inherently unreliable, the number of misses are very small compared to the available throughput received under both network loads. The reason that the packet loss during the higher network load is not monotonically increasing is attributed to the bursty nature of some of the TCP communication that was present. Since the number of misses were small, it may be feasible for a receiver to request resubmission of lost packets. Also, this shows that it may be favorable to communicate covertly over congested networks to decrease detectability because the communication is still reasonably reliable.

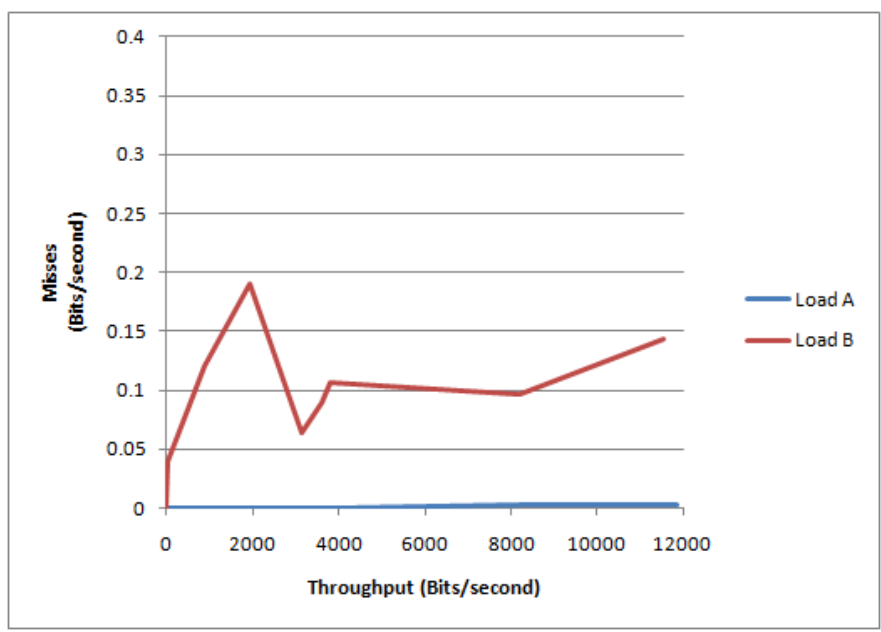

Fig. 4. Misses graphed against throughput

To determine the utility of the BCM with the given network, we measured the number of packets that were sent during both network loads (Figure 5). As mentioned earlier, the window size used during the experiments was one second. Also a maximum of six connections were considered as candidate carriers (only the six satisfied the parameters). There were other UDP connections used for controlling the media sessions, but only the connections with audio data had suitable randomness values. As a result, the maximum number of packets that were sent each second was six. This is observed in the graph. A high degree of randomness is expected because the packets contain speech audio. When the threshold parameter values are higher, the number of packets used decreases. This is a good indication that the audio data differed. Some connections had higher levels of randomness due to the fact that in some dialogs, the speakers were more verbal. If the speakers talked less, there was more silence, which translated to less randomness. Although the same connections were used as covert carriers during both network loads, there are slight differences in the values. This is because the sender and receiver were started while background traffic was already running. The randomness threshold were reached at different times depending on the network traffic present during the instantiation of the sender and receiver. 


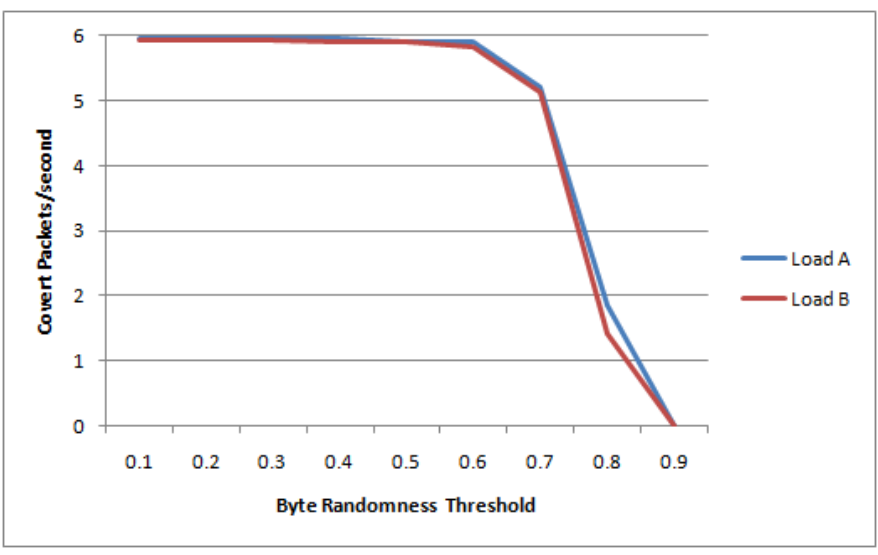

Fig. 5. Packets received graphed against Byte Rate Thresholds

\section{Vi. CONClusions And Future Work}

We have developed a novel method for secure communication that utilizes multiple concurrent covert channels and can scale as network congestion increases. Evaluation of the method shows that the communication is works with limited data loss. We have also demonstrated how the method can be configured to balance between throughput and detectability.

For future work we will determine the effects of higher throughput on quality of service (e.g., distortion, delay, etc.). In addition, we will investigate whether it is more beneficial to hide covert data based on byte similarity as in [13]. We will also evaluate the performance of the method using different network configurations such as WLAN, multicast, and switches configured with promiscuous ports. Lastly we will look into automatic methods for tuning the parameters in Table I in real time depending on network characteristics.

\section{ACKNOWLEDGMENTS}

The authors would like to thank Brenda Medina, Juan Ulloa, and Dan Landin for their valuable comments and suggestions.

\section{REFERENCES}

[1] B. Lampson, "A note on the confinement problem," Communications of the ACM, vol. 16, no. 10, pp. 613-615, 1973.

[2] A. Baliga and J. Kilian, "On covert collaboration," in Proceedings of the 9th workshop on Multimedia \& security. ACM, 2007, pp. 25-34.

[3] W. Mazurczyk and Z. Kotulski, "Covert channel for improving VoIP security," Advances in Information Processing and Protection, pp. 271280, 2008.

[4] T. Calhoun, R. Newman, and R. Beyah, "Authentication in 802.11 LANs using a covert side channel," in Communications, 2009. ICC'09. IEEE International Conference on. IEEE, 2009, pp. 1-6.

[5] S. Zander, G. Armitage, and P. Branch, "A survey of covert channels and countermeasures in computer network protocols," Communications Surveys \& Tutorials, IEEE, vol. 9, no. 3, pp. 44-57, 2007.

[6] N. Lucena, G. Lewandowski, and S. Chapin, "Covert channels in IPv6," in Privacy Enhancing Technologies. Springer, 2006, pp. 147-166.

[7] K. Szczypiorski and W. Mazurczyk, "Steganography in IEEE 802.11 OFDM symbols," Security and Communication Networks, 2011.

[8] K. Szczypiorski, "A performance analysis of HICCUPS-a steganographic system for WLAN," Telecommunication Systems, pp. 1-5, 2009.

[9] W. Mazurczyk and J. Lubacz, "LACK-a VoIP steganographic method," Telecommunication Systems, pp. 1-11, 2010.

[10] P. Fogla, M. Sharif, R. Perdisci, O. Kolesnikov, and W. Lee, "Polymorphic blending attacks," in Proceedings of the 15th USENIX Security Symposium, 2006, pp. 241-256.

[11] F. Yarochkin, S. Dai, C. Lin, Y. Huang, and S. Kuo, "Towards adaptive covert communication system," in 2008 14th IEEE Pacific Rim International Symposium on Dependable Computing. IEEE, 2008, pp. 153159.

[12] Y. Huang, B. Xiao, and H. Xiao, "Implementation of covert communication based on steganography," in International Conference on Intelligent Information Hiding and Multimedia Signal Processing. IEEE, 2008, pp. $1512-1515$.

[13] H. Tian, K. Zhou, H. Jiang, Y. Huang, J. Liu, and D. Feng, "An adaptive steganography scheme for voice over IP," in Circuits and Systems, 2009. ISCAS 2009. IEEE International Symposium on. IEEE, 2009, pp. 2922 2925.

[14] J. Rice, Mathematical statistics and data analysis. Thomson Learning, 2006.

[15] J. Acosta and N. Ward, "Achieving rapport with turn-by-turn, userresponsive emotional coloring," Speech Communication, 2010. 